\title{
Participación comunitaria, cambios urbanos: el caso de la colonia Flor del Campo, Distrito Central
}

\author{
Héctor Darío Murillo Arambú ${ }^{1}$ \\ Ana Corina Hernandez ${ }^{2}$
}

\section{RESUMEN}

En la actualidad el crecimiento de las zonas urbanas es tema de agenda a nivel mundial, situación que conlleva a la generación de nuevos espacios y demandas por parte de las personas que habitan en ellos. La sociedad urbana con la incorporación del movimiento social, genera dentro del concepto actor y su territorio, coyunturas que forjan la reacción de necesidades individuales al contexto colectivo, permitiendo volcar la lluvia de ideas para la búsqueda de solventar las necesidades existentes. Los actores sociales dentro de la Colonia Flor del Campo permiten visualizar el desarrollo de las relaciones sociales enmarcadas al ámbito urbano; durante los treinta y cinco años de fundación de la colonia Flor del Campo, el escenario urbano se ha dibujado a lo largo del tiempo, dándonos cambios urbanos en los cuales solo el tiempo y sus actores han sido testigos de este. Conocer nuestra propia situación en materia de participación comunitaria no es cuestión de simple información o curiosidad, se trata de ver como ese factor pasa a formar parte de la toma de conciencia sobre los problemas y elementos que son obstáculo para el desarrollo individual o grupal en materia de comunidad. El objetivo general del estudio es conocer la participación comunitaria como un factor determinante en los procesos de cambios urbanos desde su inicio hasta su conformación, desarrollando objetivos específicos como el conocimiento de las modalidades de participación y de la organización social-participación comunitaria. Los actores claves tomados en cuenta son: adultos mayores que han vivido en la colonia por más de 30 años, y las actividades entre líderes de la comunidad y los jóvenes. La metodología empleada fue de un enfoque de investigación cualitativo, utilizando un paradigma interpretativo, la observación y el levantamiento de encuestas semiestructuradas.

Palabras claves: desarrollo urbano, participación comunitaria, organización social, cambios urbanos, actores sociales.

\footnotetext{
${ }^{1}$ Beneficiario de beca de estudiante de posgrado de la DICYP, Posgrado latinoamericano en trabajo social, UNAH: hd.murillo.a@live.com

${ }^{2}$.Asesora, Posgrado Latinoamericano en Trabajo Social, UNAH: platsunah@yahoo.es
} 


\section{ABSTRACT}

At the present time, the growth of urban zones is a topic of worldwide agenda, a situation that brings forward the generation of new spaces and demands on behalf of the persons who inhabit in them. The urban society along with the incorporation of the social movement, generates conjunctures that forge the reaction of individual needs for the collective context, allowing a brainstorm of ideas for the search of settlement between the existing needs. The social actors inside the "Colonia Flor del Campo" visualize the development of social relations framed in an urban area; during thirty five years of foundation of this colony, the urban stage has showed itself throughout time. To know our own situation as a community participation matter is not a question of simple information or curiosity, it is a question of seeing as how this factor passes to form part of consciousness on the problems and elements that are an obstacle for the individual development or grupal development of the community. The general aim of the study was to evaluate the community participation as a determinant factor in the process of urban changes from its beginning up to its conformation, developing specific aims as knowing levels of participation and the social organization of community participation. The key actors taken in account are: adults who have lived in the colony for more than 30 years, and the activities among leaders of the community and young persons. The methodology used was a qualitative approach of investigation, using an interpretive paradigm, observation and the use of semistructured surveys.

Keywords: urban development, community participation, social organization, urban change, actors social. 


\section{INTRODUCCIÓN}

Según Castell (1995), las sociedades deben centrar sus cambios de acuerdos a sus necesidades y al entorno en que habitan, razón por la cual nos señala que: "Emerge una forma social y espacial... No es la ciudad de las tecnologías de la información profetizada por los futurólogos. Ni es la tecnópolis totalitaria denunciada por la nostalgia del tiempo pasado. Es la ciudad de nuestra sociedad" (p.19). Es por esto que el presente artículo tiene como finalidad dar a conocer cómo la participación comunitaria es un factor de cambios urbanos, investigación que tuvo lugar en la Colonia Flor del Campo, ubicada en el municipio del Distrito Central, Tegucigalpa; para lo cual fue fundamental considerar la base contextual en materia urbana y conceptual, la que se desarrolló mediante la estructuración de tres grandes categorías: sociedad urbana, desarrollo-participación y organización y actores sociales.

La participación comunitaria es un factor clave para el desarrollo de los cambios urbanos dentro de la Colonia Flor del Campo, esta participación orientada desde una perspectiva de gestión, ha logrado durante los treinta y cinco años de fundación de la colonia, impulsar desde esta la ejecución de obras por parte de instituciones públicas en materia de urbana.

La organización social ha permitido establecer un conjunto de propuestas dentro de la comunidad, que orientan la búsqueda de transformación social dentro de lo local, el involucramiento de las personas de la comunidad en organizaciones de carácter social, cultural, político y económico, enriquece la posibilidad de participar dentro de la comunidad y esta, a su vez, globalizar en el entorno local la satisfacción de necesidades y el impulso de cambios urbanos.

Con ayuda a los datos obtenidos, se lograron resultados que enfocan a un punto central, el objetivo general planteado como investigador: conocer si la participación comunitaria es un factor determinante en los procesos de cambios urbanos desde el asentamiento a la conformación de la Colonia Flor del Campo.

\section{MÉTODOS Y TÉCNICAS}

La participación comunitaria y los cambios urbanos desde el asentamiento a la colonia Flor del Campo, con el objetivo de comprender la temática planteada, será 
desarrollada desde el paradigma interpretativo del enfoque de investigación cualitati va; utilizando el método fenomenológico, el cual: "Tiene como foco entender el significado que tienen los eventos (experiencias, actos...) para las personas que serán estudiadas" (Maykut y Morehouse, 1994, p.11).

Se utilizó la temporalidad transversal, que según Sampieri, Fernández y Baptista (2004), cuando se recolectan datos en un solo momento su propósito es describir categorías y analizar su incidencia e interrelacionarlos en un momento dado; esto de forma diacrónica sobre la línea del tiempo, desde el asentamiento en la Colonia Flor del Campo del año 1979 a 2013.

Se aplicaron instrumentos como entrevistas semiestructuradas y fichas de observación; las primeras fueron aplicadas a informantes claves líderes de la comunidad, adultos mayores y jóvenes que forman parte de alguna organización dentro de la comunidad. Para establecer la muestra se hizo un primer acercamiento con autoridades dentro de la comunidad (patronato, centro de salud, iglesia) y utilizando la técnica de bola de nieve se pudo precisar la selección de personas claves (personas que forman parte de organizaciones y personas que viven desde hace más de 30 años en la colonia), con un punto de saturación para dar por finalizado con saturación teórica. También se aplicaron entrevistas semiestructuradas a personas fuera de esta rango, con la salvedad de que formaran parte de una organización o hayan sido participes (actualmente 0 en el pasado) de algún proceso en los cambios urbanos dentro su colonia. Paralelamente se aplicaron las fichas de observación que fueron estructuradas por áreas: infraestructura, servicios básicos, educación e instituciones; lo anterior permitió concluir sobre nuestra investigación.

Para el procesamiento de los datos se realizó una clasificación de estos, las entrevistas se codificaron con ayuda de software Atlas. Ti y se identificarán las categorías pertinentes en una etapa posterior para realizar el análisis de resultados.

\section{METODOLOGIA}

Para desarrollar el presente artículo fue de vital importancia contar con información base en materia urbana y social desde un contexto internacional, hasta llegar al caso específico de la Colonia Flor del Campo, permitiendo ampliar la base de la investigación en la temática de participación comunitaria y cambios urbanos. 


\section{Marco contextual}

Según información obtenida en el sitio oficial de las Naciones Unidas (2007), con su última actualización en junio de ese año, se encontró la siguiente información de interés para el desarrollo de esta investigación desde el contexto internacional:

En 1900, una de cada 10 personas vivía en ciudades. Actualmente, casi 3,000 millones de personas, o sea, casi la mitad de la humanidad, residen en centros urbanos, y ya hay 23 ciudades, 18 de ellas pertenecientes al mundo en desarrollo, con más de 10 millones de habitantes. Uno de los resultados de este rápido aumento de la población urbana es que millones de pobres de todo el mundo viven hacinados en barrios de tugurios y asentamientos ilegales, en unas condiciones de vida por debajo de los niveles mínimos necesarios para garantizar la salud de las familias y comunidades (párr.1).

Uno de los procesos que se resaltan en cuando al crecimiento de los espacios urbanos, es la labor de los gobiernos locales para gestionar proyectos; según el informe de la CEPAL (2003), los gobiernos locales aparte de ejecutar proyectos eficaces: "Adolecieron en sus gestiones de varios de los defectos de las empresas públicas nacionales de servicios, de modo que la ola privatizadora los encontró en condiciones de plantear opciones diferentes" (p.186); lo que conlleva a la concretización de estrategias en la búsqueda de otras alternativas en materia del desarrollo urbano y asentamiento humano.

El escenario urbano, Honduras también presenta datos relevantes sobre la temática, dentro de las estadísticas demográficas: cuenta con una población estimada de 8 , 535,692 millones de habitantes según la encuesta EPHPM (2013) y con una tasa de crecimiento natural de $1.9 \%$. La pirámide poblacional indica que la mayoría de los habitantes son jóvenes y los grupos etarios se reducen según aumenta la edad, en donde los adolescentes menores de 19 años representan el $43.8 \%$ de la población; en cuanto a la población de la tercera edad, representada por personas de 60 años y más, conforman una minoría de apenas un $9.5 \%$.

La población total por sexo es de 4, 099,703 hombres, que representan un $48.0 \%$ y 4, 435,988 mujeres, que representa un $52 \%$. Según el INE (2013), la población urbana es de 3,986,354 habitantes y de esta, el Distrito Central concentra aproximadamente el $27.6 \%$ con 1,101,942 habitantes; mientras que San Pedro Sula con 676,959 habitantes representa el $17.0 \%$. 
Tegucigalpa, Francisco Morazán, cuenta con una extensión territorial que abarca los $8.619 \mathrm{~km}$, ubicada en el centro del país entre los $13^{\circ} 40^{\prime}$ y $15^{\circ} 02^{\prime}$ de Lat $\mathrm{N}$ y los $86^{\circ}$ $43^{\prime}$ y $87^{\circ} 39^{\prime}$ de Long 0 .

La Alcaldía Municipal del Distrito Central (AMDC) en 2007, señala el diagnóstico de acciones para Tegucigalpa y su desarrollo sustentable, en el cual nos dice que Tegucigalpa para:

El año 2004, el Distrito Central tenía una población total de 874, 515 habitantes, de la cual el $46.3 \%$ correspondió a hombres y el $53.7 \%$ a mujeres y representaba el $12.5 \%$ de la población total del país y el $27.5 \%$ de la urbana. La población se caracteriza por ser joven, dado que el $45.5 \%$ es menor de 18 años; seguido por la comprendida entre los 19 y 49 años que conforma el $41 \%$; y la mayor de 50 años el $13.5 \%$ (p. 9).

En los últimos 25 años, el área urbana de Tegucigalpa triplicó su tamaño. La mayor parte de la expansión ocurrió desde mediados de 1970 y finales de 1980. Impulsada por el rápido crecimiento de la población, el área urbana aumentó de 2,360 hectáreas en 1975 a 6,020 hectáreas en 1987 y 8,360 hectáreas en 2000. En términos porcentuales, ese cambio representó un aumento del $155 \%$ y $39 \%$, previamente a 1987 y 2000, respectivamente (...) la densidad de población disminuyó de 134 a 99 hectáreas por persona (p.12).

\section{Colonia Flor del Campo}

El Distrito Central cuenta con un crecimiento de sus áreas urbanas cada día mayor. Según el Programa de Administracion de Tierras de Honduras (PATH, 2004), la Colonia Flor del Campo fue fundada en agosto de 1979, siendo reconocida por la municipalidad el 5 de diciembre de ese mismo año fungiendo como alcalde Elvin Santos, para este tiempo la Colonia abarcaba un perímetro de 79.31 manzanas aproximadamente.

La Colonia Flor del Campo colinda al norte con la Colonia Modesto Rodas Alvarado, al sur con la Colonia Luís Andrés Zúniga, al este con la Colonia La Pradera y al oeste con Vía Los Laureles. Se individualizó en predios con un área aproximada de 240 metros cuadradas, los que da predios de 12 × 20 metros; con un aproximado de 9,900 habitantes, cuenta con 3 sectores con un área de 79.31 manzanas, con un total de viviendas de 1,921 según los recibos del agua (PATH, 2004). 
Actualmente, según el Sistema Unificados de Registros (2014) de la página oficial del Instituto de la Propiedad, el total de viviendas es de 2,107 basándose en el último registro de la Dirección General de Catastro y Geografía, en el cual se incluyen las agrupaciones o divisiones de los lotes de terrenos.

\section{Perspectiva conceptual}

Dado que esta investigación está enfocada en dos ejes, cambio urbano y participación comunitaria, se tomarán como líneas bases fundamentos socioculturales de la participación comunitaria, mismos que constituyen una mirada desde la teoría y la práctica a los elementos socioculturales que distinguen y condicionan al proceso participativo en el contexto comunitario.

Para el desarrollo de esta investigación se toma la teoría de la acción social planteada por Weber (1944), que parte de la pretensión de fundar una sociología comprensiva, es decir, una: “...Ciencia que pretende 'interpretándola [i.e. interpretar]', la acción social para de esa manera explicarla en sus desarrollos y efectos..." (p.5). Para ello, este autor define a la acción social como cualquier tipo de proceder humano que significativamente: "...Se orienta por las acciones de otros, las cuales pueden ser presentes o esperadas como futuras..." (Weber, 1944, p.16). Con base en lo anterior, el desarrollo de este marco conceptual tomará como base los aportes teóricos de Manuel Castells en materia urbana y otros autores que fortalecen la temática de participación comunitaria, organización social y sus actores.

\section{Sociedad urbana}

Según Castells (1972), la sociedad urbana está conformada por una estructura y organización social, misma que se encuentra envuelta en la generación de cambios mediante la participación de sus actores según las necesidades individuales, colectivas y las provocadas por las condiciones de sus entornos (culturales, étnicos-culturales, políticas y territoriales).

Para Castells (1973), una sociedad es una estructura urbana con una forma de organización social, siendo esta la que asegura las actividades productivas y de los medios de consumo de la comunidad en general, esto da una idea de las fortalezas de una organización dentro de una comunidad y su participación como gestor de cambio urbano; pero qué sucede con este concepto en la actualidad, es claro que las bases teóricas trascienden a lo largo del tiempo, a pesar de esto la sociedad se encuentra en constante evolución y los conceptos de sociedad urbana son desarrolla- 
dos para la temática de ciudad.

Manuel Castells (1974) conduce a la dignidad del habitar como fundamento del ser del hombre que comienza con la propia construcción y engloba una dimensión del mismo ser, pues habitar no es alojarse, no es una función accidental del hombre, sino una de sus manifestaciones esenciales, llevando esta situación como uno de los procesos necesarios para el desarrollo. Asimismo, Castells (1986) dice que: "...Gracias únicamente a la permisividad del Estado, pueden contar los ocupantes ilegales con la base especial necesaria para su existencia cotidiana..." (p. 295). Señalando claramente el papel importante que juega el Estado en las condiciones de los asentamientos, así como otra organización que permita lo mismo.

Los movimientos sociales urbanos probablemente se deben a que: "Por definición el concepto de movimiento social como agente de transformación social es impensable en la teoría marxista" (Castells, 1986, p.29). Pero, además Castells descubre y descifra una ideología propiamente urbana que capta los modos y las formas de la organización social e implica una cultura urbana como oposición a la rural, que consagra el paso decisivo de una forma comunitaria a otra asociativa (Castells, 1974).

Castells (1995) analiza la existencia de un conjunto de transformaciones articuladas históricamente que implican al capitalismo como sistema social:

Al informacionalismo entendido como el modo de desarrollo en el que la información substituye a la mano de obra como factor determinante como modelo de desarrollo y a las tecnologías de la información como poderosos instrumentos de trabajo. La perspectiva teórica en la que se basa es que las sociedades están organizadas en función de procesos humanos estructurados por las relaciones de producción (acción ejercida por el hombre sobre la materia para obtener de ella un producto que le beneficie), experiencia (acción de los seres humanos sobre si mismos en el marco biológico y cultural para la satisfacción de sus necesidades y deseos) y poder (relación de los seres humanos que sobre la base de la producción y de la experiencia impone la voluntad de unos individuos sobre otros por medio del uso potencial o real de la violencia) históricamente determinadas (p. 9).

Dentro de este mismo marco es importante desarrollar el concepto de comunidad, el cual significa que: "Todas las formas de relación que se caracterizan por un elevado grado de intimidad personal, profundidad emocional, compromiso moral, cohesión social y continuidad en el tiempo... puede encontrarse en... localidad, religión, nación, raza, profesión o (causa común). Su arquetipo... es la familia" (Nisbet, 1966, p.47-48), 
asimismo, una comunidad es un grupo global que cuenta con dos principales características: "(1) Lugar donde el individuo puede encontrar la mayor parte de las actividades y experiencias, que le son importantes. (2) El grupo está unido entre sí, por un sentido compartido de la posesión, así como por un sentimiento de identidad" (Broom \& Selznick, 1968, p.31).

Para Henri Lefebvre (1971): "La ciudad proyecta sobre el terreno una sociedad, una totalidad social o una sociedad considerada como totalidad, comprendida su cultura, instituciones, ética, valores, en resumen sus superestructuras, incluyendo su base económica y las relaciones sociales que constituyen su estructura propiamente dicha" (p. 40), esto nos lleva a formarnos una idea de los lasos que se pueden lograr dentro de estructuras sociales, mismas que pueden fortalecerse según los roles que cada individuo juega y el medio en que se desenvuelve.

Según Borja (1988), la participación es: “...Sobre todo el encuentro entre las instituciones representativas, partidos y administración, por una parte, y los movimientos y organizaciones sociales que existen autónomamente de los partidos políticos y de los mecanismos participativos..." (p. 27).

Esto permitió como investigación abordar el trabajo de campo, lo que generó una investigación con hallazgos en cuanto a la temática estudiada y en especial en la Colonia Flor del Campo.

\section{ANÁLISIS Y RESULTADOS}

Con el objetivo de obtener información cualitativa de los procesos de participación comunitaria en los cambios urbanos en la Colonia Flor del Campo, se han desarrollado dos ejes: la participación comunitaria a través de treinta y cinco años de crecimiento urbano y comunidad, sociedad y su organización. Para el primero de estos, la Colonia Flor del Campo fundada en el año de 1979 ha sido testigo de cambios en materia urbana desde sus inicios con el asentamiento de los primeros pobladores de forma informal, que ha permitido en la actualidad la existencia de 2,107 viviendas divididas entre los 3 sectores, en los que se pueden encontrar centros de educación, centro de salud, posta policial, instituciones u organizaciones comunales y sociales, vías de acceso y servicios básicos; estos con una morfología de espacio ortogonal que deriva de la construcción de viviendas u obras de forma perpendicular a las calles. 
Esto permite como investigador ver más allá de la simple obra civil; la relación de estas con la participación de la comunidad en la búsqueda de entornos que faciliten la incorporación de un sector especifico con su alrededor, buscando el mejoramiento de la calidad de vida de sus pobladores. Mediante la vinculación con los datos obtenidos en la práctica se encuentra información relevante que permite conocer las condiciones urbanas de la colonia con los primeros pobladores que la habitaron, estos afirman que: "Aquí cuando nosotros entramos en la Colonia Flor del Campo se empezaron con unas poquitas habitaciones y aquí la suerte que todas las dirigencias de los dirigentes que han estado... han venido haciendo su parte que les ha correspondido y si lo llevo hacer un recorrido aquí de lo que es Flor del Campo, usted se dará cuenta de los trabajos, de proyectos de arreglo de calle, del servicio de agua, electricidad, bueno acá tenemos todo, una colonia completa con todos los servicios" (informante 1). Los cambios urbanos son visibles y los informantes los han ratificado, por tal razón, la recolección de información secundaria es básica para contrastar la información de nuestras entrevistas; por tal razón, se verificó en instituciones públicas como el SANAA, FHIS, IP, SOPTRAVI y AMDC, la ejecución de proyectos por parte de estas, obteniendo información de más de 40 proyectos de infraestructura en la Colonia Flor del Campo durante los últimos 35 años.

Otra vinculación sobre la línea del tiempo desde la mirada de líderes de organizaciones y adultos mayores que habitan en la Colonia con respecto a los cambios urbanos, al referirse a estos desde una perspectiva del tiempo, manifiestan que: "Cuando yo vine a la Colonia solo habían caminos, ahora apertura de calles pavimentadas, el asunto de las viviendas eran de madera, aún de pedazos de lata y cartón, en cuanto a vivienda hay cambios, ahora son de material y bloque, no había agua, alcantarillado y alumbrado" (informante 2); lo que ayudó a crear un mapa mental de cómo era el paisaje urbano treinta y cinco años atrás.

También se descubrió durante esta investigación, con relación a la línea del tiempo, que: "Primero fue el agua, pero no le digiera potable, era aguas por sectores, después viene la luz eléctrica, después los teléfonos fijos, después lo del servicio de agua potable" (informante 3); la línea de tiempo es clara, esto nos permite establecer un punto de partida al momento de los primeros asentamientos en la Colonia Flor del Campo.

Las nuevas generaciones manifiestan otra visión del tiempo con respecto a los procesos de cambios urbanos, por ejemplo, al realizar la misma interrogante sobre los cambios urbanos que recuerda en su Colonia dicen: "...Acá arriba el Centro Israel cambiaron la calle, la pavimentaron toda, está cambiando mucho" (informante 4); 
dando referencia de proyectos urbanos que han sido realizados en los últimos 2 o 3 años en la Colonia.

Las etapas de participación a lo largo de la historia en la Colonia Flor del Campo han sido parte gestora del impulso social para el desarrollo de cambios urbanos que permiten mejorar las condiciones de vida de sus pobladores, siendo esta la que asegura las actividades productivas y de los medios de consumo de la comunidad en general, la instalación de servicios de agua potable, alcantarillado público, aguas residuales, electricidad y telefonía; esto da la idea de las fortalezas de una organización dentro de una comunidad y su participación para impulsar estos cambios, dándole el significado de "colonia con todos los servicios", identificando que dentro de un espacio en donde existe una población y organización, hay actividades en búsqueda de la generación de cambios dentro de su entorno.

La participación comunitaria ha sido base para impulsar cambios urbanos en la Colonia Flor del Campo. Con base en el trabajo de campo se logró comprender cómo esta participación ha formado parte del desarrollo desde el asentamiento en el año de 1979, hasta formar parte de las colonias del Distrito Central en la actualidad; según lo desarrollado en la sección de participación y sus actores del marco teórico, la Colonia cuenta con actores ligados a la toma de decisiones en el caso de las autoridades del patronato y junta de agua, como gestores principales para impulsar cambios urbanos en la comunidad; asimismo, los ligados a la acción del terreno, siendo estos los pobladores de la Colonia Flor del Campo, resaltando la importancia de la interacción de los actores para generar desarrollo local.

Con respecto al presente eje, buscar la relación entre la organización social y participación comunitaria como factor que genera cambios urbanos, es importante para el desarrollo de esta investigación, más cuando la vinculación de la persona en su espacio pasa de ser un individuo a formar parte de un colectivo.

Durante el proceso de contrucción a través de la información obtenida en campo, se destaca la forma en la cual la comunidad canaliza mediante comités auxiliares las necesidades de su sector, las cuales son dadas a conocer a las autoridades patronales, como lo dice este informante: "Una comunidad si no está organizada no avanza, por ejemplo aquí nosotros hemos hecho varios proyectos... entonces lo que hacemos nosotros, organícense en comités auxiliares, entonces aquí a la fecha tenemos varios comités auxiliares" (informante 1), de esta forma las autoridades gestionan con otras instancias solventar las solicitudes. 
Se destaca en lo expresado por otra persona entrevistada en calidad de líder de la comunidad, el uso del término "estatus de la Flor del Campo como una colonia modelo", la participación activa por parte de los pobladores de la Colonia en la organización de comités, lo que permite generar procesos más eficientes dentro de la misma, uno de ellos es el cobro del servicio de agua potable, para ello en las oficinas de la Junta de Agua de la Colonia Flor del Campo, existe un sistema computarizado con el SANAA, en el cual los usuarios pueden hacer el pago del servicio en estas oficinas sin necesidad de salir del perímetro de la Colonia. Según un informante, un ejemplo es que el SANAA los ha tomado como base para la generación de otros proyectos comunitarios que permitan ofrecer mejores opciones a los usuarios de este vital servicio.

Contar con una organización social que permite canalizar las necesidades individuales y colectivas de una forma más eficiente, está relacionada con la participación que se encuentra entorno al ámbito de gestionar a las instancias respectivas las necesidades de la comunidad e involucrarse en actividades que se desarrollen con esta, por ejemplo: "La instalación del proyecto de agua potable, cuando se formaron los comités, entonces ellos colaboraron para cuestión de incentivar a la comunidad" (informante 9).

Desde esta parte, la comunidad no trabaja directamente en la ejecución de obras, sino como actor indirecto en las mismas, sin menospreciar la importancia de esto: "La formación de comités sectoriales y por cuadra, haciendo labores y gestionando ayudas" (informante 2); la organización por sectores se encuentra conformado por hombres y mujeres adultas, con excepción de los grupos juveniles; resulta que no existe diferencia entre los informantes claves al relacionar la organización a los comités sectoriales o también conocidos como auxiliares.

\section{CONCLUSIONES}

1. La participación comunitaria ha forjado las bases para la gestión de proyectos urbanos en la Colonia Flor del Campo, durante el tiempo transcurrido desde su fundación en el año de 1979 a la actualidad; la evidencia de proyectos urbanos son claros con el registro de cuarenta y dos (42) proyectos dentro de la Colonia que brindan a sus pobladores mejoramiento de la calidad de vida y comunicación con un circuito denominado "ciudad". 
2. La participación de los actores dentro del territorio local de la Colonia Flor del Campo ha permitido estructurar dentro de la misma una serie de organizaciones que buscan satisfacer necesidades de sus pobladores; lo que permite canalizar en el contexto local la demanda generada dentro del perímetro de la Colonia.

3. La participación comunitaria es un factor clave para el desarrollo de los cambios urbanos dentro de la Colonia Flor del Campo, esta participación orientada desde una perspectiva de gestión, ha logrado durante los treinta y cinco años de fundación de la Colonia impulsar desde esta la ejecución de obras por parte de instituciones públicas en materia de urbanización.

4. La sociedad urbana con la incorporación del movimiento social, genera dentro del concepto actor-territorio, coyunturas que forjan la reacción de necesidades individuales al contexto colectivo, permitiendo la búsqueda de soluciones a necesidades existentes. Los actores sociales dentro de la Colonia Flor del Campo permiten visualizar el desarrollo de las relaciones sociales enmarcadas en el ámbito urbano; durante los treinta y cinco años de fundación de la Colonia, el escenario urbano se ha dibujado a lo largo del tiempo, produciendo cambios urbanos de los cuales solo el tiempo y sus actores son testigos.

5. Basados en la información secundaria recolectada durante los treinta y cinco de fundación de la Colonia Flor del Campo desde 1979, los cambios urbanos han sido desarrollados, a pesar de ellos, estos cambios no ha sido constantes a lo largo de las casi cuatro decadas de fundación, siendo la decada de 2000, con la ejecucion de veinte (20) proyectos registrados, el período con la mayor cantidad de proyectos urbanos.

6. La organización social ha permitido establecer un conjunto de propuestas dentro de la comunidad, que orientan la búsqueda de transformación social dentro de lo local, el involucramiento de las personas de la comunidad en organizaciones de carácter social, cultural, político y económico, que enriquece las posibilidad de participar dentro de la comunidad y esta a su vez globalizar en el entorno local la satisfacción de necesidades y el impulso de cambios urbanos.

7. La organización social es un factor importante para la concretización de soluciones a las necesidades de la comunidad, la Colonia Flor del Campo ha desarrollado estructuras organizativas llamadas comités auxiliares, término utilizado por los informantes claves; mismos que son localizados por sectores y tienen el objetivo de canalizar las solicitudes realizadas por parte de las demás personas de la zona 
para resolver problemas en su sector; estos comités están encargados de llevar las solicitudes al nivel de las autoridades patronales u otra organización.

8. La participación comunitaria y organización social basadas en las personas de la comunidad no varía en grandes rasgos a pesar de ser estos líderes adultos mayores y jóvenes; las ideas fundamentales de estas dos variables con respecto al término de cambios urbanos, son similares; los grupos identifican obras civiles en momentos específicos de sus vidas, a pesar de ello no se puede dejar fuera que por parte de los adultos mayores existe una biblioteca mental de procesos significativos durante sus vidas.

9. Uno de los puntos débiles es la falta de apropiación por parte de los jóvenes de los proyectos que han marcado pauta en las condiciones de vida en las que ellos se están desarrollando; razón por la cual es imprescindible que los generaciones adultas y líderes de la comunidad impulsen programas y proyectos que busquen ese encuentro de identidad local que todos los pobladores de la Colonia Flor del Campo deben de poseer y de esta forma empoderarse de cada uno de los proyectos que se ejecutan en su comunidad, dándole un valor relevante en el desarrollo sus vidas.

10.Dentro de los factores que condicionan a la comunidad existe el factor "lo que hacen los actores" según lo señala Pirez (2005); esto hace la diferencia del porqué las personas no relacionan los proyectos con las obras y al momento de vincular el eje de organizaciones, las personas crean una conexión con las obras de las cuales han sido testigos, dejando de lado los proyectos que otras organizaciones ejecutan en su misma comunidad.

11.La participación comunitaria es un factor importante para el desarrollo del entorno urbano de la Colonia Flor del Campo, a pesar de ello, con el trabajo de campo finalizado no se logró dar respuesta al supuesto hipotético de si los hombres participan más que las mujeres. 


\section{AGRADECIMIENTOS}

A todo el personal del Posgrado Latinoamericano en Trabajo Social y a la Dirección de Investigación Cientifica y Posgrados de la UNAH, por apoyar esta investigación con una beca; a mí madre Alba Arambú, padre Héctor Murillo, hermanos, sobrinos y Guillermo Raudales.

\section{BIBLIOGRAFÍA}

Alcaldía Municipal del Distrito Central. (2007). Acciones para Tegucigalpa y su desarrollo sustentable. Recuperado de: http://www.capital450.hn/diagnostico.php Borja, J. (1988). Participación. ¿Para qué? Revista Urbana, II, 25-44. Broom, L. \& Selznik, P. (1968). Sociology: A Test with Adapted Readings. Nueva York: Harper \& Rowe.

Castells, M. (1995). La ciudad informacional. Tecnologías de la información, estructuración económica y el proceso urbano-regional. Madrid: Alianza Editorial.

Castells, M. (1973). Imperialismo y urbanización en América Latina. España: Alianza Editorial.

Castells, M. (1974). La cuestión urbana. España: Alianza Editorial.

Castells, M. (1986). La ciudad y las masas. España: Alianza Editorial.

Castells, D. (2007). Otro concepto de desarrollo. En Contribuciones a la Economía. Comisión Económica para América Latina y el Caribe. (2003). Gestión urbana para el desarrollo sostenible en América Latina y el Caribe. Santiago de Chile.

Instituto Nacional de Estadística. (2013). Cuadragésima cuarta (XLIV) encuesta permanente de hogares de propósitos múltiples. Honduras. Recuperado de: http://www.ine.gob.hn

Lefebvre, H. (1971). De lo rural a lo urbano. Primera Edición. Paris, Francia: Anthropos Editorial.

Maykut, P. \& Morehouse, R. (1994). Beginning Qualitative Research. A Philosophic and Practical Guide. London: The Falmer Press.

Nisbet, R. (1966). The Sociological Tradition. Nueva York: Basic Books.

Sampieri, R.; Fernández, C. y Baptista, P. (2004). Metodologías de la investigación. México: McGraw-Hill Interamericana.

Weber, M. (1944). Economía y sociedad. México-Buenos Aires: Fondo de Cultura Económica. 
